\title{
The Application of Image Retrieval Technology based on Visual Features in Agricultural Informatization
}

\author{
Jianfang Cao ${ }^{1,2, *}$, Qingshan Zhao ${ }^{1}$ and Lichao Chen ${ }^{3}$ \\ ${ }^{1}$ Xinzhou Teachers University in China; ${ }^{2}$ Taiyuan University of Technology in China; ${ }^{3}$ Taiyuan University of Science \\ and Technology in China
}

\begin{abstract}
At present, the agriculture images are increasing greatly day by day. It becomes increasingly difficult for users to find out the desired images. Building the database to store images and realizing the automatic retrieval of images is very important in agriculture field. Based on the study of visual feature retrieval technology of images, this article puts forward a new retrieval algorithm using color, texture and shape features according to the characteristics of crop image database. The retrieval accuracy rate has been improved greatly and by reasonably setting the feature library, the retrieval efficiency is greatly improved. An experimental system is constructed through programming. We establish a relatively complete test database for crop images and generic image database, demonstrate the effectiveness of the proposed retrieval algorithm. Finally, the retrieval performance using different features is evaluated and compared, and the problems and future works are analyzed.
\end{abstract}

Keywords: Agricultural informatization, feature extraction, image retrieval, visual feature.

\section{INTRODUCTION}

The $21^{\text {st }}$ century is the era of information agriculture, and agricultural information technology is the key driving force to the high-speed, healthy and sustainable developing agriculture. Agricultural information technology [1] is an edge applied discipline in which agriculture image information retrieval technology based on visual features is one of the important components. Image retrieval based on visual features [2] refers to various characteristic retrievals according to image contents, and it can find images which contain the specified characteristics or specific content directly from the database. For the agricultural information system, the storage organization and retrieval of information are the main problems to be solved in most cases. As the mature of mass storage technology and the decline of cost, information storage is no longer the main concern, but the need to find out the needed information quickly from the vast amounts of information resources becomes the urgent issue.

As an exploratory attempt of the image retrieval system, the experiment system of this article provides the following functions: realizing the visual index interface; implementing the offline index and online query functions respectively; acquiring the sample images by category view, and then processing the retrieval, in which the retrieval of comprehensive features such as color, texture and shape is achieved.

\section{VISUAL FEATURE EXTRACTION}

Image visual features [3] are the original features or attributes of images. The basic image features are the natural features which the human vision can determine such as regional brightness, contour, color or texture, shape etc. Visual image feature extraction is the basis of image retrieval.

\subsection{Color Feature}

Compared with geometrical features, color has certain stability and strong robustness to change of noise, size, resolution and direction [4]. In most cases, color is the most simple and effective feature for description of images and the resolution of people's eyes to color images is much higher than that to black and white images. So the information color images contain is much more than gray images and color feature extraction is very important for image retrieval.

\subsubsection{Color Quantization Space}

RGB color, consisting of red, green and blue components, is the commonly used color space [5]. Currently a variety of images use RGB color model and it is supported on most physical devices. However, the studies have found that the RGB components are not independent of each other and there exists a correlation to some degree. But HSV color space has good independence property of visual perception and it may feel continuously change from all types of color components, that is to say, the color change which can be perceived by the human eyes can be measured by the Euclidean distance between the color components. Therefore this article adopts HSV color space.

\subsubsection{Sub-block Thought}

The color histogram only reflects the distribution information of image color and loses the spatial distribution information of color. In order to get the spatial distribution information of color, we often segment images. Since image 
segmentation algorithms are complicated and the segmentation result is not very ideal, the scholars often use the subblock. Frequently-used block strategy is dividing the image uniformly into $\mathrm{m}^{*} \mathrm{n}$ blocks. But this method can't emphasize the middle part of the main body of the image. It is easy to divide the main body into different blocks but it and undermines its integrity. Most important of all, it does not consider the relationship between blocks. Therefore, this article uses a new sub-block method of image based on overlap blocks. It is shown in Fig. (1).

\begin{tabular}{|l|l|l|l|}
\hline $\mathrm{B}_{11}$ & $\mathrm{~B}_{12}$ & $\mathrm{~B}_{13}$ & $\mathrm{~B}_{14}$ \\
\hline $\mathrm{B}_{21}$ & $\mathrm{~B}_{22}$ & $\mathrm{~B}_{23}$ & $\mathrm{~B}_{24}$ \\
\hline $\mathrm{B}_{31}$ & $\mathrm{~B}_{32}$ & $\mathrm{~B}_{33}$ & $\mathrm{~B}_{34}$ \\
\hline $\mathrm{B}_{41}$ & $\mathrm{~B}_{42}$ & $\mathrm{~B}_{43}$ & $\mathrm{~B}_{44}$ \\
\hline
\end{tabular}

Fig. (1). Image sub-block thought.

The steps are as follows:

(1) The image is divided into $4 \times 4$ sub-blocks.

where: $\mathrm{A}=\left\{\mathrm{B}_{11}\right\}, \mathrm{B}=\left\{\mathrm{B}_{14}\right\}, \mathrm{C}=\left\{\mathrm{B}_{41}\right\}, \mathrm{D}=\left\{\mathrm{B}_{44}\right\}, \mathrm{E}=\left\{\mathrm{B}_{12}, \mathrm{~B}_{13}\right.$, $\left.\mathrm{B}_{22}, \mathrm{~B}_{24}\right\}, \mathrm{F}=\left\{\mathrm{B}_{32}, \mathrm{~B}_{33}, \mathrm{~B}_{42}, \mathrm{~B}_{43}\right\}, \mathrm{G}=\left\{\mathrm{B}_{21}, \mathrm{~B}_{22}, \mathrm{~B}_{31}, \mathrm{~B}_{32}\right\}, \mathrm{H}=\left\{\mathrm{B}_{23}\right.$, $\left.\mathrm{B}_{24}, \mathrm{~B}_{33}, \mathrm{~B}_{34}\right\}, \mathrm{I}=\left\{\mathrm{B}_{22}, \mathrm{~B}_{23}, \mathrm{~B}_{32}, \mathrm{~B}_{33}\right\}$.

The method makes the center part of the image surrounded 3 times and 4 angles of the image surrounded 1 time, which not only makes sure that the integrity of the image is maintained but also stresses the main body of the image to a certain degree.

(2) Assign different weights $\omega_{n}$ for different image blocks. Because the weights directly influence the accuracy of the algorithm, the weights should be decided combining with the characteristics of image. The center part of image is usually the main part of the image and is assigned the higher weight; edge part is assigned the smaller weight. In this article, the weight is determined according to the method of area division. First, count the number $C_{i j}$ of sub-blocks of each overlapped block when overlapping blocks; Second, compute the total number $C=\sum_{i, j}^{4} C_{i j}$ of appeared sub-blocks; Finally, compute the total of number $C_{k}(k=A, B, \mathrm{~L}, I)$ of sub-blocks of each overlapped block; Then the weight is $\omega_{n}=C_{n} / C$.

\subsubsection{Color Feature Extraction}

This article extracts the main color as image color feature. The main color is the color that appears frequently in the image. The process is as follows:

(1) Segment the image according to sub-block thought;

(2) Compute color histogram for each sub-block, and find the color that occupies the largest pixel area in color histogram as the main color of sub-block, and take it as the color feature of the sub-block;

(3) Compute the color feature of the image according to the weight set.

\subsection{Texture Feature}

Texture feature is to compute corresponding statistical values from the texture image and quantify the gray level features of internal texture [6]. Texture feature reflects local structure of the image.

\subsubsection{Image Edge Feature Extraction}

Edge detection is important for image understanding because the edge is the boundary of the image and is the significant change part of the local image change. Many experiments have shown the Canny operator is an optimal edge detection operator. First, two edge components, $G_{x}(x, y)$ and $G_{y}(x, y)$, are generated and then the amplitude and edge orientation is calculated using the following formula (1) and (2) respectively. Next, the important edges of the gradient image, obtained by comparing a threshold value $T_{1}$ are extracted. Finally, the edge histogram is quantized uniformly into $n$ segments $\angle G_{1}, \angle G_{2}, \cdots, \angle G_{n}$. The results of the edge direction are shown in Fig. (2).

$$
\begin{aligned}
& |G|=\sqrt{G_{x}^{2}(x, y)+G_{y}^{2}(x, y)} \\
& \angle G=\arctan \left(G_{x}(x, y) / G_{y}(x, y)\right)
\end{aligned}
$$

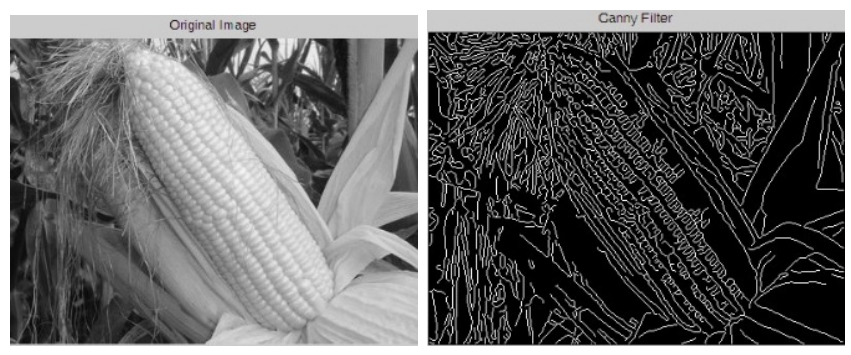

Fig. (2). An example of edge detection using Canny operator.

\subsubsection{Texture Feature Extraction}

This article uses two-dimension wavelet decomposition method of wavelet transform to obtain image texture feature. In order to describe the texture features of an image, five commonly used gray-level statistical features along with four different angles $(0,45,90$, and 135$)$ are presented as follows. Energy is the local homogeneity measure of images. For the homogeneous regions, when the particular pixel pairs are more leading to larger energy values. Homogeneity describes the degree of the texture thickness and gray uniformity, highvalue array element which concentrates near the main diagonal have large values. Contrast shows the amount of the local change and describes the degree of the groove depth of the texture.

(1) Energy:

$E N G=\sum_{i=0}^{N-1} \sum_{j=0}^{N-1} P^{2}(i, j \mid d, \theta)$

(2) Contrast:

$C O N=\sum_{i=0}^{N-1} \sum_{j=0}^{N-1}(i-j)^{2} P(i, j \mid d, \theta)$ 
(3) Entropy:

$E N T=-\sum_{i=0}^{N-1} \sum_{j=0}^{N-1} P(i, j \mid d, \theta) \log (P(i, j \mid d, \theta))$

(4) Homogeneity:

$H O M=\sum_{i=1}^{N-1} \sum_{j=1}^{N-1} P(i, j \mid d, \theta) /\left[1+(i-j)^{2}\right]$

(5) Max probability:

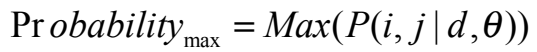

where, $P(i, j \mid d, \theta)$ is probability of the occurrence of every pixel having a gray level $i$ and $j$ giving the space distance $d$ and the direction $\theta$, and is often expressed in matrix form.

\subsection{Shape Feature}

When people see an image, what they note first is the object including the image. And the shape feature is the most basic and meaningful feature except for color and texture features. Therefore, extracting shape feature for images has important significance for image retrieval. The moment is the important representation of the overall shape of images [3]. Some key features of 2D images are directly related to the moment, such as the image size, centroid and rotation, and so on. Zernike moment is the orthogonal function based on Zernike polynomial, which has the advantages of invariance of image rotation and strong anti-noise ability. It is applied to extract shape feature of image in this article. The formula of computing Zernike moment $Z_{n m}$ (plural) is:

$C_{n m}=\frac{2 n+2}{N^{2}} \sum_{r=1}^{N / 2} R_{n m}(\rho) \sum_{\sigma=1}^{8 r} \cos \frac{\pi m \sigma}{4 r} f(r, \sigma)$

$S_{n m}=\frac{2 n+2}{N^{2}} \sum_{r=1}^{N / 2} R_{n m}(\rho) \sum_{\sigma=1}^{8 r} \sin \frac{\pi m \sigma}{4 r} f(r, \sigma)$

Among them: $C_{n m}$ is the real part of Zernike moment $Z_{n m}$, $S_{n m}$ is the imaginary part of Zernike moment $Z_{n m}, n$ is the positive integer or zero, $m$ is the positive integer or the negative integer $(n-|m|$ is even and $n \geq|m|), R_{n m}(\rho)$ is the radial polynomial of pixel point $(x, y)$ of the image $f(x, y)$, $r=\max (|x|,|y|), \rho=2 r / N(N \times N$ is the size of the image $f(x, y))$.

$$
\sigma= \begin{cases}\frac{2(r-x) y}{|y|}+\frac{x y}{r} & \text { if }|x|=r \\ 2 y-\frac{x y}{r} & \text { if }|y|=r\end{cases}
$$

\section{THE REALIZATION OF SYSTEM FUNCTION}

We use Visual Basic 6. 0 to program an image retrieval experimental system based on visual features, and use SQL databases as background to store images and their corresponding feature information. This system divides into two sub-systems which are the subsystem of image and feature storing and the subsystem of image retrieval. Fig. (3) shows the interface of image and feature storing subsystem and Fig. (4) shows the interface of image retrieval subsystem.

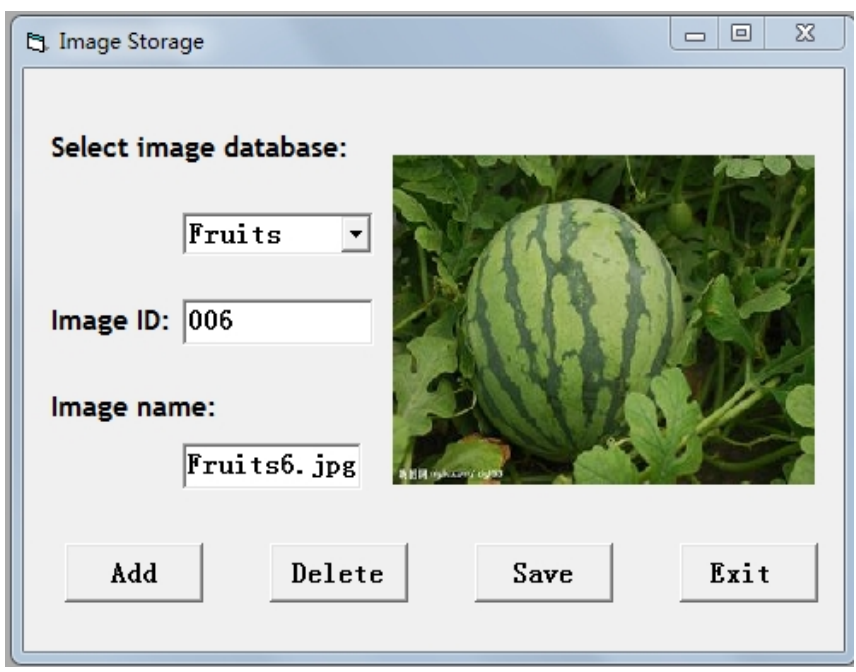

Fig. (3). The interface of image and feature storing subsystem.

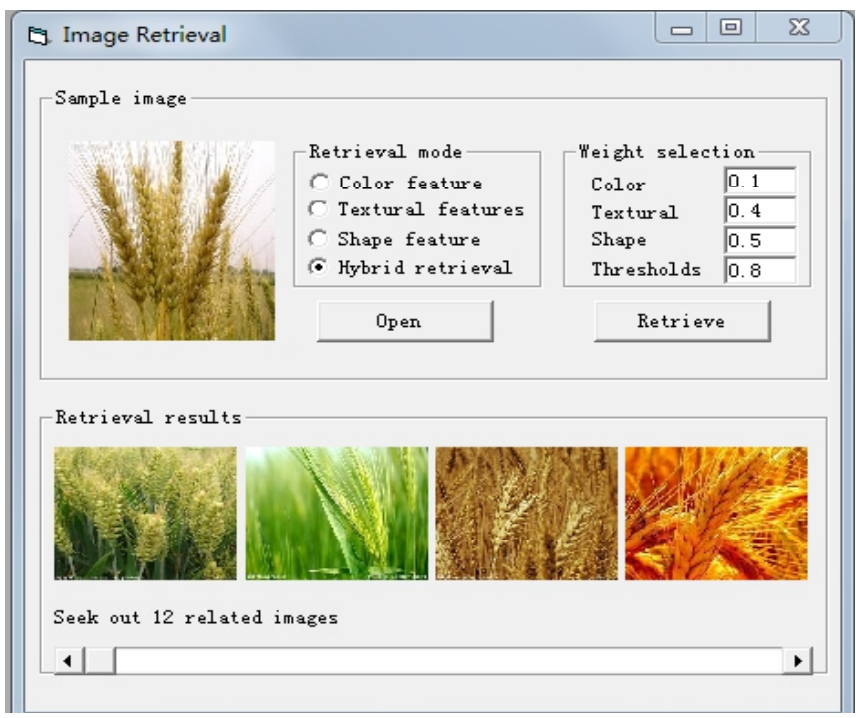

Fig. (4). The interface of image retrieval subsystem.

The system currently provides 4 classes and nearly 500 images for users to browse and query. After logging in the system, we can successively open the retrieval image, choose the way to retrieve, set the weight of color, texture and shape features and choose appropriate threshold according to the steps provided by system. And after we clicks the "search" button, the system first would extract the features of sample image, and then make matching calculation with the characteristics set of the index database, and finally the retrieval results are exhibited in the bottom result column.

\section{EXPERIMENT AND RESULT ANALYSIS}

Currently accuracy rate, recall rate and the retrieval speed are often adopted to reflect the retrieval performance of the algorithm $[7,8]$. Among them, the average accuracy rate is the most representative. This article mainly focuses on discussing the average precision rate. Supposing that $a$ is num- 
ber of the correctly retrieved images, $b$ is number of the false-retrieved images, accuracy rate is $a /(a+b)$.

Fig. (5) is a sample image and Fig. (6) is the retrieval results when we choose "Hybrid retrieval" and the weights are set as follows: color weight is 0.5 , texture weight is 0.1 , shape weight is 0.4 and threshold is 0.8 .

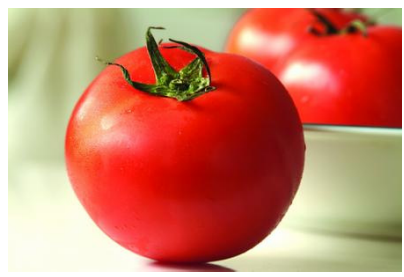

Fig. (5). The sample image.

In order to reflect the performance of the system more clearly, we calculate the average accuracy rate for 4 types of images when using single feature and hybrid retrieval. The results are shown in Table $\mathbf{1}$.

From the experiment results, we can obviously see, the retrieval method combined with the characteristics could get a good retrieval performance, of which the average accuracy rate is about $60 \%$. The performance of hybrid retrieval is better and the average accuracy rate could reach about $80 \%$. In fact, color feature only represents the statistical distribution of all sorts of colors in the image, and do not contain any color spatial local information. And image texture feature is a kind of regional feature, which can be taken as a recurring local patterns of image and their arrangement rules, and the content of the texture characterization can be seen as a spatial information distribution message. In a word, texture feature can be used as a supplement description of the distribution state of color and combining two features is better than one feature alone when retrieving. Similarly, in many cases adopting three features to make a comprehensive retrieval can obtain better performance.

\section{CONCLUSION AND FUTURE WORKS}

In this article, we proposed an image retrieval method based on visual features and applied the method in the agriculture field. The article gathered three features to retrieve and we can choose different weight value according to different needs. Through tests, the proposed method can achieve good retrieval performance. In the future, we will also improve the image feature extraction algorithm, especially the feature extraction method, to make it reflect the discipline of each point of image in the spatial distribution. In addition, we think that the introduction of clustering idea in data mining plays a vital role in large and super large image database retrieval, especially when combined with related field technology, which will promote the standardization description of multimedia database.

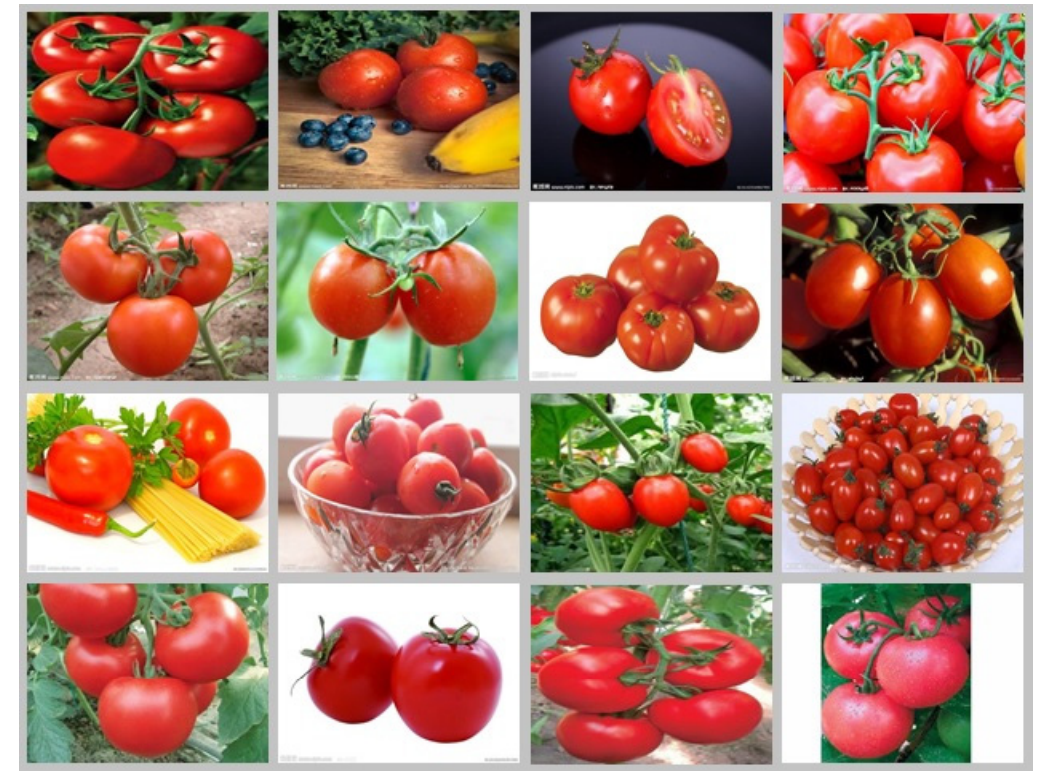

Fig. (6). Retrieval results of the sample image.

Table 1. Comparasion of the Average AccuracyRate.

\begin{tabular}{|c|c|c|c|c|}
\hline Category & Color & Texture & Shape & Hybrid \\
\hline \hline vegetables & $59.1 \%$ & $53.2 \%$ & $61.3 \%$ & $75.9 \%$ \\
\hline fruits & $62.3 \%$ & $61.0 \%$ & $65.1 \%$ & $80.2 \%$ \\
\hline crops & $60.7 \%$ & $58.4 \%$ & $60.1 \%$ & $73.6 \%$ \\
\hline flowers & $66.9 \%$ & $62.5 \%$ & $64.8 \%$ & $83.1 \%$ \\
\hline
\end{tabular}




\section{CONFLICT OF INTEREST}

The authors confirm that this article content has no conflict of interest.

\section{ACKNOWLEDGEMENTS}

This work was supported by National Natural Science Foundation of China under Grant No. 61202163 and by the Natural Science Foundation of Shanxi Province under Grant No.2012011011-5 and No. 2013011017-2 and by the Technology Innovation Project of Shanxi Province under Grant No. 2013150 and by the Key disciplines supported by Xinzhou Teachers University under Grant No. XK201308.

\section{REFERENCES}

[1] Q. H.Yu, Y. H. Li, "Study on actuality and strategy of land fragmentation in middle China based on GIS," Int. Conf. Elect. Cont. Eng., Wu Han, June 2010.
[2] W. M. Smeulders, M. Worring, S. Santini, A. Gupta, R. Jain, "Content-based image retrieval at the end of the early years", IEEE Trans. Pattern Anal. Mach. Intell. (TPAMI), vol. 22, no. 12, pp. $3845,2000$.

[3] K. Yu, Y. Lin, and J. Laffert,.” Learning image representations from the pixel levelvia hierarchical sparse coding", In: Proceedings IEEE Conf. Comp. Vision and Pattern Recognit., pp. 1713-1720, 2011.

[4] K. E. A. van de Sonde, T. Gevers, and C. G. M. Snoek, "Evaluating color descriptors for object and scene recognition," Pattern Anal. Mach. Intell., vol. 32, no. 9, pp. 1582-1596, 2000.

[5] T. Tuytelaars, C. Schmid, "Vector quantizing feature space with a regular lattice," In: Proc. IEEE Int. Conf. Comp. Vision (ICCV), pp. $1-8,2007$.

[6] S. Gao, I. W. H. Tsang, L.-T. Chi a, and P. Zhao, "Local features are notlonely Laplacian sparse coding for image classification," IEEE Comp. Soci. Conf. Comput. Vision and Pattern Recognit., pp. 3555-3561, 2010.

[7] T. F. Yang, J. Ma, S. Gao and C. R. Cui, "Spatial tower matching for image classification," J. Comput. Inform. Syst., vol. 8, no. 16, pp. 6757-6764, 2012.

[8] T. F. Yang, J. Ma, "Unsupervised Object Localization with Latent Dirichlet Allocation," In: Proc. Int. Conf. Comp Sci. Artif. Intell., pp. 230-235, 1996.

Received: September 22, 2014

Revised: November 30, 2014

Accepted: December 02, 2014

(C) Cao et al.; Licensee Bentham Open.

This is an open access article licensed under the terms of the Creative Commons Attribution Non-Commercial License (http://creativecommons.org/licenses/by-nc/3.0/) which permits unrestricted, non-commercial use, distribution and reproduction in any medium, provided the work is properly cited. 